\title{
VARIABILIDADE ESPACIAL DA FORÇA DE DESPRENDIMENTO DE FRUTOS DO CAFEEIRO
}

\author{
GABRIEL A. e S. FERRAZ ${ }^{1}$, FÁBIO M. DA SILVA ${ }^{2}$, MARCELO S. DE \\ OLIVEIRA $^{3}$, FLAVIO C. DA SILVA ${ }^{4}$, RAFAEL DE L. BUENO ${ }^{5}$
}

\begin{abstract}
RESUMO: O objetivo deste trabalho foi utilizar técnicas de georreferenciamento e de geoestatística para analisar a variabilidade espacial da força de desprendimento de frutos de cafeeiros por meio de semivariogramas e pela interpolação por krigagem. O trabalho foi conduzido no município de Três Pontas - MG, Brasil. A força de desprendimento dos frutos verdes e de cerejas dos cafeeiros foi obtida por meio de um protótipo de dinamômetro em pontos georreferenciados. A dependência espacial dos dados foi analisada por meio de ajustes de semivariogramas, clássico e robusto, para o método dos mínimos quadrados ordinários e ponderados, e apenas o ajuste clássico, para os métodos da máxima verossimilhança e máxima verossimilhança restrita. Testaram-se, para cada um dos métodos, os modelos esférico, exponencial e gaussiano. Os mapas de isolinhas obtidos por krigagem foram produzidos, baseados no melhor método e modelo de ajuste da função semivariograma, que foram obtidos pelas estatísticas de validação. As variáveis em estudo apresentaram estruturas de dependência espacial, as quais foram modeladas pelos semivariogramas, o que possibilitou a confecção dos mapas de isolinhas de distribuição espacial, obtidos por krigagem. Foi possível identificar os locais mais propícios para se iniciar a colheita seletiva e mecanizada dos frutos do cafeeiro.
\end{abstract}

PALAVRAS-CHAVE: Face de exposição solar, agricultura de precisão, geoestatística, colheita mecanizada e seletiva, café.

\section{SPATIAL VARIABILITY OF THE DETACHMENT FORCE OF COFFEE FRUIT}

ABSTRACT: The aim of this study was to use the georeferencing and geostatistics techniques to evaluate the spatial variability of coffee fruit detachment force by semivariograms adjustments and kriging interpolation. The study was conducted in Três Pontas, MG, Brazil. The detachment force of green and mature coffee fruit was obtained throughout a prototype dynamometer on georeferenced locations. The spatial dependence data was evaluated by classical and robust semivariogram adjustments, using ordinary least square and weighted least squares methods. Maximum likelihood and restricted maximum likelihood methods were also evaluated, but only for the classical semivariogram. Spherical, exponential and Gaussian models were compared for all the evaluated methods of semivariogram estimation. The isoline maps obtained by kriging were generated based on the best adjustment method and model of the semivariogram function obtained by the statistical validation. The studied variables showed spatial dependence, which were modeled by semivariograms that allowed plot isoline maps of the spatial distribution obtained by kriging. It was possible to identify the best places to start the mechanical and selective coffee fruit harvest.

KEYWORDS: sun exposure side, precision agriculture, geostatistics, selective and mechanized harvest, coffee.

\footnotetext{
${ }^{1}$ Eng $^{\circ}$ Agrícola, Prof. Dr., Depto. de Engenharia, Universidade Federal de Lavras/Lavras - MG, Fone: (35) 3829-

4566, gabriel.ferraz@deg.ufla.br

${ }^{2}$ Eng $^{\circ}$ Agrícola, Prof. Dr., Depto. de Engenharia, Universidade Federal de Lavras/Lavras - MG, famsilva@ufla.br

${ }^{3}$ Eng $^{\circ}$ Agrícola, Prof. Dr., Depto. de Ciências Exatas, Universidade Federal de Lavras/Lavras - MG, marcelo.oliveira@dex.ufla.br

${ }^{4}$ Eng $^{\mathrm{O}}$ Agrícola, Prof. Dr., Depto. de Engenharia Agrícola e do Meio Ambiente, Universidade Federal Fluminense/Niterói -

RJ, flavio-ter@vm.uff.br

${ }^{5}$ Eng $^{\circ}$ Agrícola, CEIFA Máquinas/Lavras - MG, rafaeldelimabueno@hotmail.com

Recebido pelo Conselho Editorial em: 12-6-2012

Aprovado pelo Conselho Editorial em: 20-5-2014
} 


\section{INTRODUÇÃO}

A cafeicultura no Brasil gerou um crescimento econômico de notória relevância ao longo de sua história e possibilitou ao País destacar-se como maior produtor de café do mundo (LOPES et al., 2012). Além de ser uma cultura extremamente importante, destaca-se por apresentar elevado custo de produção, de forma que os cafeicultores estão sempre em busca de sua redução (RIBEIRO et al., 2009). De acordo com ORTEGA \& JESUS (2011), as inovações agronômicas, em conjunto com o uso mais intensivo de máquinas agrícolas, além de facilitar a aplicação de outras inovações, contribuem para o aumento de produtividade e para a racionalização das operações. Segundo SILVA et al. (2009), o uso da mecanização agrícola nas diversas operações de campo é uma das grandes ferramentas do produtor rural na redução de custos, mediante o aumento da velocidade de realização de tarefas.

De acordo com SILVA et al. (2010b), a colheita mecanizada do café é realizada por meio de varetas situadas em cilindros verticais oscilantes, que envolvem a planta de cafeeiro lateralmente, derriçando os frutos pelo efeito da vibração. SOUZA et al. (2005) descrevem que o número de ciclos necessários ao desprendimento dos frutos é proporcional ao tempo de desprendimento, e a influência significativa do estádio de maturação sobre essa variável indica que o tempo de aplicação da vibração é uma variável que deve ser considerada no processo de colheita de frutos do cafeeiro visando à colheita seletiva.

Uma das dificuldades encontradas pelos cafeicultores durante a colheita mecanizada é a definição do melhor momento para se iniciar a colheita e também a definição de uma adequada vibração e velocidade operacional da máquina. Para aumentar o desempenho operacional da colhedora de café, é necessário estabelecer alguns parâmetros. SILVA (2008) observou que a força de desprendimento dos frutos verdes era $73 \%$ maior que a dos frutos cerejas e que esta diferença poderia ser um fator importante para a colheita mecanizada seletiva dos frutos do cafeeiro. Desta maneira, realizar diferentes estudos acerca da força de desprendimento dos frutos pode contribuir para que o cafeicultor tenha um produto mais competitivo.

Segundo SILVA et al. (2008), CARVALHO et al. (2009), ALVES et al. (2009a) e SILVA et al. (2010c), a agricultura de precisão apresenta-se como um conjunto de tecnologias capaz de auxiliar o produtor rural a identificar as estratégias a serem adotadas para aumentar a eficiência no gerenciamento da agricultura, maximizando a rentabilidade das colheitas, tornando o agronegócio mais sustentável e competitivo face ao processo de globalização da economia, contribuindo assim para o sucesso da atividade agrícola.

A geoestatística é uma ferramenta importante para análise de dados no âmbito da agricultura de precisão. Com sua utilização, é possível caracterizar e modelar a variabilidade espacial das mais diversas variáveis que se deseja estudar, a fim de se identificar inter-relações destas no espaço. Sua utilização permite também estudar padrões de amostragem adequada e, além disto, possibilita a confecção de mapas temáticos que são ferramentas importantes na tomada de decisão.

Diante do exposto, o objetivo deste trabalho foi realizar a análise da distribuição espacial da força de desprendimento de frutos de cafeeiros de acordo com sua maturação e com sua face de exposição solar, empregando-se recursos da geoestatística. Por meio da utilização de diferentes métodos e modelos de semiaviograma, objetivou-se caracterizar a estrutura de variabilidade espacial da variável no fenômeno em estudo e, com a obtenção de mapas de krigagem, objetivou-se caracterizar a distribuição dos valores da variável estudada na área e na época em questão.

\section{MATERIAL E MÉTODOS}

O experimento foi desenvolvido na fazenda Brejão, localizada no município de Três Pontas, sul de Minas Gerais (coordenadas geográficas do ponto central da área são 2125'58" de latitude sul e $45^{\circ} 24^{\prime} 51 "$ de longitude oeste de Greenwich), em uma área de 22 ha de lavoura de cafeeiro (Coffea arabica L.) da cultivar Topázio, com idade de 3 anos e 7 meses, transplantada no espaçamento de 3,8 m entre linhas e 0,8 m entre plantas, totalizando 3.289 plantas ha $^{-1}$. Os pontos limítrofes da área 
foram obtidos por meio de um GPS geodésico Topcon FC-100 (Topcon Positioning Systems Inc, Livermore, Califórnia, EUA). Esse equipamento é composto por uma antena móvel, um computador portátil e antena posicionada em uma base fixa, para correção dos dados.

A coleta dos dados de força de desprendimento foi realizada em julho de 2009, em uma malha irregular contendo 48 pontos (Figura 1). Cada ponto amostral correspondeu a quatro plantas: duas plantas localizadas na rua de cafeeiros onde o ponto foi georreferenciado, e as outras duas plantas localizadas em cada rua lateral ao ponto de referência (Figura 1).

A força de desprendimento dos frutos do cafeeiro foi obtida considerando-se a face de exposição solar, uma vez que a face leste do cafeeiro recebia a maior incidência solar no turno da manhã, e a face oeste, maior incidência dos raios solares no turno da tarde. Consideraram-se, também, dois estádios de maturação dos frutos: cereja e verde.

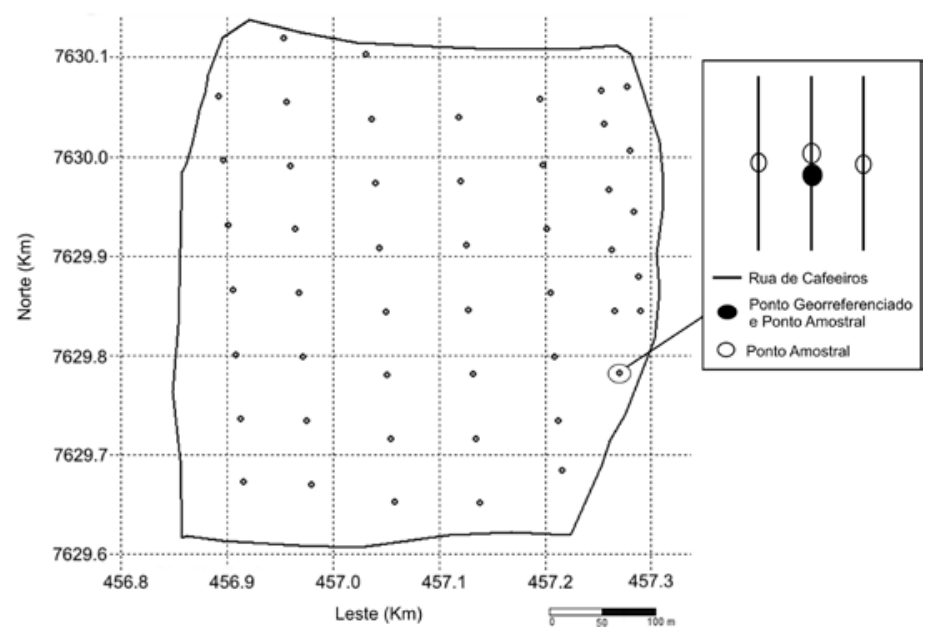

FIGURA 1 - Malha amostral irregular e detalhamento do esquema amostral. Irregular sampling grid and detail of the sampling scheme.

Para a obtenção dos dados de força de desprendimento (FD), foi realizada a coleta de cinco frutos de cada estádio de maturação, em cada face de exposição solar, totalizando vinte frutos por planta. Os frutos foram coletados três dias antes de o produtor realizar a colheita na área. Após esta medição, foi obtida a média da força de desprendimento composta pelos frutos de quatro plantas do ponto georreferenciado, diferenciando-se cada estágio de maturação e cada face de exposição solar.

Foi realizada também a junção dos dados de cada face de exposição para formar um valor médio total de força de desprendimento, em cada ponto estudado, tanto para frutos cerejas quanto para frutos verdes, encontrando a força de desprendimento em área total da planta exposta aos raios solares. Foi obtida, ainda, a diferença entre a força de desprendimento dos frutos verdes e cerejas, para as duas faces de exposição solar e em área total da planta.

A determinação desta força de desprendimento dos frutos foi realizada por meio de um dinamômetro portátil, especificamente construído para este fim. Sua construção e calibração foram realizadas no Laboratório de Protótipos, no Departamento de Engenharia da Universidade Federal de Lavras (UFLA), em Lavras - MG (SILVA et al., 2010b). Seu princípio de funcionamento baseiase na Lei de Hooke, que correlaciona a deformação de corpos, dentro do regime elástico, com a força exercida sobre o corpo, tal que a força é proporcional ao deslocamento a partir de seu ponto de equilíbrio. No caso, foi utilizada uma mola específica, determinando-se sua constante elástica em função da deformação, como se segue: 


$$
F=k \cdot \Delta l
$$

em que,

"F” é a força $(\mathrm{N})$;

"k" é a constante elástica da mola $\left(\mathrm{N} \mathrm{m}^{-1}\right)$, e

$\Delta \mathrm{l}$ é a deformação da mola $(\mathrm{m})$.

A aferição do dinamômetro foi realizada por meio da deformação elástica da mola, solicitada por corpos com massas conhecidas, previamente pesadas em balança de precisão eletrônica. A partir deste procedimento, determinou-se o valor da constante elástica da mola, obtendo-se 57,37 N $\mathrm{m}^{-1}$, possibilitando medidas de 0,00 a $12,03 \mathrm{~N}$.

A dependência espacial da força de desprendimento dos frutos de cafeeiros foi analisada por meio de ajustes de semivariogramas, clássico (Matheron) e robusto (Cressie-Hawkins), e interpolação por Krigagem ordinária. O semivariograma clássico foi estimado como se segue:

$$
\hat{\gamma}(\mathrm{h})=\frac{1}{2 \mathrm{~N}(\mathrm{~h})} \sum_{\mathrm{i}=1}^{\mathrm{N}(\mathrm{h})}\left[\mathrm{Z}\left(\mathrm{x}_{\mathrm{i}}\right)-\mathrm{Z}\left(\mathrm{x}_{\mathrm{i}}+\mathrm{h}\right)\right]^{2}
$$

em que,

$\mathrm{N}$ (h) é o número de pares experimentais de observações $\mathrm{Z}\left(\mathrm{x}_{\mathrm{i}}\right)$ e $\mathrm{Z}\left(\mathrm{x}_{\mathrm{i}}+\mathrm{h}\right)$ separados por uma distância h. O semivariograma é representado pelo gráfico $\hat{\gamma}(h)$ versus $h$.

Do ajuste de um modelo matemático aos valores calculados de $\hat{\gamma}(h)$, são estimados os coeficientes do modelo teórico para o semivariograma denominado de efeito pepita, $\mathrm{C}_{0}$; contribuição, $\mathrm{C}_{1}$; patamar, $\mathrm{C}_{0}+\mathrm{C}_{1}$; e o alcance, a, conforme descrito por BACHMAIER \& BACKES (2008).

O estimador representado na [eq. (3)], proposto por CRESSIE \& HAWKINS (1980), é mais robusto para estimar o semivariograma quando, nos dados georreferenciados utilizados na análise, há possivelmente presença de dados discrepantes (outliers).

$$
\hat{\gamma}(\mathrm{h})=\frac{1}{2} \frac{\left[\mathrm{N}(\mathrm{h})^{-1} \sum_{\mathrm{N}(\mathrm{h})} \sqrt{|(\mathrm{Z}(\mathrm{s}+\mathrm{h})-\mathrm{Z}(\mathrm{s}))|}\right]^{4}}{0.457+\frac{0.494}{\mathrm{~N}(\mathrm{~h})}}
$$

A estimação da função semivariogram a não se constitui como algo trivial encontrado na literatura, sendo possível justificar estatisticamente diferentes estratégias, embasadas em diferentes princípios e pressupostos, alguns destes de difícil verificação objetiva (MINGOTI \& ROSA, 2008). Em função dessa diversidade metodológica, para tal estimação, neste estudo, foram feitas conjugações de alternativas em duas etapas de estimação: (i) Na primeira etapa, escolheu-se qual ou quais estimadores seriam usados na estimação das semivariâncias (consideraram-se dois estimadores possíveis: o estimador clássico de Matheron (MA) e o estimador robusto de CressieHawkins (CH)); (ii) Na segunda etapa, o ajuste dos modelos para a função semivariograma foi feito utilizando diferentes métodos de estimação (consideraram-se quatro métodos possíveis: o método dos quadrados mínimos ordinários (OLS); o método dos quadrados mínimos ponderados (WLS); o método da máxima verossimilhança (ML), e o método da máxima verossimilhança restrita (REML). Conjugaram-se então as seguintes seis combinações: (MA, OLS), (MA, WLS), (CH, OLS), (CH, WLS), (MA, ML), (MA, REML), todas estas combinações foram aplicadas como implementadas no software utilizado. Em cada uma das seis combinações, utilizaram-se três modelos tradicionais 
diferentes (esférico, exponencial e gaussiano), totalizando então dezoito ajustes diferentes. Como critério de qualidade de ajuste, optou-se pela validação, critério já proposto na literatura geoestatística e amplamente utilizado. Desta maneira, a conjugação que obteve as melhores estatísticas de validação foi a conjugação escolhida.

FARACO et al. (2008), avaliando a qualidade do ajuste dos modelos teóricos espaciais, segundo diferentes métodos de ajuste do semivariograma, concluíram que o método da Validação foi o mais adequado para a escolha do melhor ajuste do modelo do semivariograma, resultando em mapas temáticos mais acurados. Este critério também foi utilizado por JOHANN et al. (2010) \& FERRAZ et al. (2012) para a escolha dos melhores ajustes de semivariograma. Sendo assim, neste trabalho, a validação foi o critério utilizado para a escolha dos métodos e modelos de ajuste do semivariograma.

De acordo com ISAAKS \& SRIVASTAVA (1989), a validação é a técnica de avaliação de erros de estimativa que permite comparar valores preditos com os amostrados. $\mathrm{O}$ valor da amostra, em certa localização Z(si), é temporariamente descartado do conjunto de dados e, então, é feita uma previsão por krigagem no local $\hat{Z}\left(s_{(i)}\right)$, usando-se as amostras restantes. Desta forma, é possível retirar alguns valores que serão muito úteis para a escolha do método, como o Erro Médio (EM), o Desvio- Padrão dos Erros Médios (DP $\mathrm{EM}_{\mathrm{M}}$ ), o Erro Médio Reduzido (ER) e o Desvio-Padrão do Erro Médio Reduzido $\left(\mathrm{S}_{\mathrm{ER}}\right)$. Assim, o Erro Médio por validação cruzada (EM) é obtido pela seguinte expressão:

$$
\mathrm{EM}=\frac{1}{\mathrm{n}} \sum_{\mathrm{i}=1}^{1}\left(\mathrm{Z}\left(\mathrm{s}_{\mathrm{i}}\right)-\hat{\mathrm{Z}}\left(\mathrm{s}_{(\mathrm{i})}\right)\right)
$$

em que,

$n$ é o número de dados;

$\mathrm{Z}\left(\mathrm{s}_{\mathrm{i}}\right)$, valor observado no ponto $s_{i}$,

e $\hat{Z}\left(s_{(i)}\right)$ valor predito por krigagem ordinária no ponto $s_{i}$, sem considerar a observação $Z\left(s_{i}\right)$ (FARACO et al., 2008).

Segundo CRESSIE (1993), o erro médio reduzido (ER), desvio-padrão dos erros médios $\left(D_{\mathrm{EM}}\right)$ e desvio-padrão dos erros médios reduzidos $\left(\mathrm{S}_{\mathrm{ER}}\right)$ podem ser utilizados para avaliar os modelos. O erro médio reduzido (ER) é definido pela equação:

$$
E R=\frac{1}{n} \sum_{i=1}^{n} \frac{Z\left(s_{i}\right)-\hat{Z}\left(s_{(i)}\right)}{\sigma\left(\hat{Z}\left(s_{(i)}\right)\right)}
$$

em que,

$\sigma\left(\hat{Z}\left(s_{(i)}\right)\right)$ é o desvio-padrão da krigagem no ponto $s_{i}$, sem considerar a observação $Z\left(s_{i}\right)$.

O desvio-padrão dos erros médios reduzidos $\left(\mathrm{S}_{\mathrm{ER}}\right)$ é obtido a partir de:

$$
S_{E R}=\sqrt{\frac{1}{n} \sum_{i=1}^{n}\left\{\frac{Z\left(s_{i}\right)-\hat{Z}\left(s_{(i)}\right)}{\sigma\left(\hat{Z}\left(s_{(i)}\right)\right)}\right\}^{2}}
$$

A diferença média entre os valores será mais próxima de zero quanto melhor for a estimativa. Os critérios de escolha baseados na validação devem encontrar os valores de EM e de ER mais próximos de zero, o valor $\mathrm{DP}_{\mathrm{EM}}$ deve ser o menor possível e o valor de $\mathrm{S}_{\mathrm{ER}}$ deve ser o mais próximo de um. 
Além do critério de validação, foram analisados a variância e o patamar, pois de acordo com WEBSTER \& OLIVER (2007), o valor do patamar deve ser próximo do valor da variância dos dados, se a nuvem de pontos do semivariograma apresenta patamar. Semivariogramas com patamar, além de atenderem à hipótese intrínseca, também atendem à hipótese de estacionariedade de segunda ordem. Sob esta hipótese, a variância da variável estudada é estimada tanto pela variância amostral dos dados quanto pelo patamar do semivariograma ajustado. A verificação objetiva destas hipóteses, por um teste estatístico, por exemplo, ainda não possui uma solução proposta na literatura, restando apenas a aceitação de suas plausibilidades pelo exame dos semivariogramas ajustados (uma nuvem de pontos de semivariâncias para a qual se pode ajustar um modelo teórico de semivariogram, é indicativo de atendimento da hipótese intrínseca, e uma nuvem de pontos com patamar indica atendimento da hipótese de estacionariedade de segunda ordem).

Após o ajuste dos semivariogramas e da escolha da conjugação que proveu as melhores estatísticas de validação, foi realizada a interpolação dos dados por krigagem ordinária, de forma a possibilitar a visualização dos padrões de distribuição espacial das variáveis na lavoura.

Para a análise geoestatística e para a confecção dos mapas, foi utilizado o sistema computacional estatístico $\mathrm{R}$ por meio de sua biblioteca geoR (RIBEIRO JÚNIOR \& DIGGLE, 2001). Os mapas foram gerados com coordenadas Universal Transversa de Mercator (UTM) na zona 23K, na qual está inserida a região de Três Pontas.

\section{RESULTADOS E DISCUSSÃO}

A análise descritiva da força de desprendimento dos frutos cerejas dos cafeeiros expostos ao sol na face leste (FDC Leste), expostos na face oeste (FDC Oeste), expostos em área total da planta (FDC Total) e a força de desprendimento dos frutos verdes de cafeeiro expostos ao sol na face leste (FDV Leste), expostos na face oeste (FDV Oeste) e expostos em área total da planta (FDV Total) são apresentadas na Tabela 1. Nessa tabela, ainda são apresentados os valores da diferença entre a força de desprendimento dos frutos verdes e cerejas total (DFD Total), na face oeste (DFD oeste) e na face leste (DFD leste).

TABELA 1. Estatística descritiva das variáveis força de desprendimento de frutos cerejas, verdes e a diferença entre verdes e cerejas em planta total, no lado leste e oeste da planta (N) em 2009. Descriptive statistics to the variables detachment force of mature and green fruit and difference between green and mature in hole plant, in the east side and west side of the plant $(\mathrm{N})$ in 2009

\begin{tabular}{cccccccccc}
\hline & Mín & Máx & Média & Mediana & DP & Var & CV & k & Assimetria \\
\hline FDC Total & 4,91 & 8,36 & 6,87 & 6,85 & 0,91 & 0,84 & 13,32 & $-0,83$ & $-0,07$ \\
FDC Leste & 4,95 & 8,43 & 6,99 & 7,09 & 0,92 & 0,85 & 13,20 & $-0,80$ & $-0,45$ \\
FDC Oeste & 4,36 & 8,73 & 6,74 & 6,74 & 1,06 & 1,12 & 15,72 & $-0,61$ & $-0,01$ \\
\hline FDV Total & 9,34 & 10,96 & 10,15 & 10,19 & 0,35 & 0,12 & 3,44 & 0,01 & -0.22 \\
FDV Leste & 9,14 & 10,93 & 10,14 & 10,23 & 0,43 & 0,18 & 4,22 & $-0,61$ & $-0,25$ \\
FDV Oeste & 9,18 & 11,03 & 10,15 & 10,15 & 0,42 & 0,17 & 4,11 & $-0,20$ & $-0,05$ \\
\hline DFD Total & 1,20 & 5,26 & 3,28 & 3,24 & 0,90 & 0,81 & 27,51 & $-0,37$ & 0,09 \\
DFD Leste & 0,91 & 5,08 & 3,16 & 3,00 & 0,94 & 0,88 & 29,76 & $-0,19$ & 0,12 \\
DFD Oeste & 1,46 & 5,67 & 3,41 & 3,21 & 1,05 & 1,11 & 30,89 & $-0,64$ & 0,21 \\
\hline
\end{tabular}

FDC - força de desprendimento de frutos cerejas; FDC - força de desprendimento de frutos verdes (FDV); DFD - diferença entre a força de desprendimento de frutos verde e cereja; Mín - valor mínimo da variável; Máx - valor máximo da variável; DP - desviopadrão; Var - variância; CV - coeficiente de variação; k- coeficiente de curtose

A FDC leste apresentou valores variando de 4,95 $\mathrm{N}$ a 8,43 $\mathrm{N}$ e valores médios de 6,99 $\mathrm{N}$; para a FDC oeste, os valores variaram de $4,36 \mathrm{~N}$ a $8,73 \mathrm{~N}$ e média de $6,74 \mathrm{~N}$, e para a força de desprendimento independente da face (FDC Total), os valores variaram de 4,91 $\mathrm{N}$ a 8,36 $\mathrm{N}$ e com valor médio de $6,87 \mathrm{~N}$. A FDV leste variou de $9,14 \mathrm{~N}$ a $10,93 \mathrm{~N}$ e valor médio de $10,14 \mathrm{~N}$; para a 
FDV oeste, os valores variaram de 9,18 $\mathrm{N}$ a 11,03 $\mathrm{N}$ com valores médios de 10,15 $\mathrm{N}$, e a força de desprendimento dos frutos verdes, independentemente da face de exposição solar (FDV Total), variou de 9,34 $\mathrm{N}$ a 10,96 $\mathrm{N}$ e com valor médio de 10,15 $\mathrm{N}$. A DFD leste variou de 0,91 N a 5,08 N; para DFD oeste, variou de 1,46 N a 5,67 N, e para a DFD total, a variação foi de 1,20 N a 5,26 N.

A partir da análise dos valores mínimos e máximos, e também da média da força de desprendimento dos frutos do cafeeiro, foi possível verificar que existe variação nos dados, porém somente o conhecimento desta amplitude não é suficiente para saber quais são os locais onde se encontram os maiores valores de força de desprendimento e os locais onde se encontram as menores forças para o desprendimento dos frutos de cafeeiros. Desta forma, é necessário utilizar ferramentas geoestatísticas para que se possa modelar a variabilidade espacial dos dados, bem como realizar a confecção de mapas de isolinhas que possibilitem o gerenciamento das intervenções necessárias.

Baseado na metodologia de análise geoestatística, foi possível quantificar a magnitude e a dependência espacial da força de desprendimento de frutos cereja e verde expostos ao sol na face leste, oeste e independente da face de exposição, bem como da diferença entre a força de desprendimento dos frutos verde e cereja nas mesmas condições de exposição solar (Tabela 2 e Figura 2).

TABELA 2. Métodos, modelos e parâmetros estimados dos semivariogramas experimentais para as variáveis força de desprendimento de frutos cereja (FDC), verde (FDV) e a diferença entre verde e cereja (DFD), de acordo com a face de exposição solar (Leste e Oeste) e em área total de exposição, no ano de 2009. Methods, models and parameters estimated by the experimental variogram to the variables detachment force of mature fruit (FDC), green fruit (FDV) and difference between green and mature (DFD) according to the solar exposure side (East and West) and in the hole area of exposure in 2009.

\begin{tabular}{lccccccccccc}
\hline & Método & Modelo & $\mathbf{C}_{\mathbf{0}}$ & $\mathbf{C}_{\mathbf{1}}$ & $\mathbf{C}_{\mathbf{0}}+\mathbf{C}_{\mathbf{1}}$ & $\mathbf{A}$ & $\mathbf{a}$ & $\mathbf{E M}$ & $\mathbf{D P}_{\text {EM }}$ & ER & $\mathbf{S}_{\text {ER }}$ \\
\hline FDC Total & WLS - R & Esf & 0,00 & 0,86 & 0,86 & 139,51 & & 0,0116 & 0,6006 & 0.0091 & 0.8357 \\
FDC Leste & WLS - R & Exp & 0,00 & 0.86 & 0.86 & 43,18 & 129,35 & 0,0136 & 0,7234 & 0,0084 & 0,8578 \\
FDC Oeste & OLS - C & Esf & 0,00 & 1,11 & 1,11 & 154,18 & & 0,0167 & 0.6447 & 0,0125 & 0,8551 \\
\hline FDV Total & WLS - R & Esf & 0,00 & 0,12 & 0,12 & 99,92 & & 0,0016 & 0,3414 & 0,0031 & 1,0599 \\
FDV Leste & ML & Exp & 0,17 & 0,01 & 0,18 & 33,03 & 98,94 & 0.0001 & 0.4419 & 0.0001 & 1.0209 \\
FDV Oeste & WLS - R & Esf & 0,00 & 0,17 & 0,17 & 102,32 & & -0.0048 & 0.3852 & -0.0064 & 0,9852 \\
\hline DFD Total & OLS - C & Exp & 0,00 & 0,81 & 0,81 & 66,94 & 200,55 & -0.0059 & 0.6976 & -0.0045 & 0.9549 \\
DFD Leste & WLS - C & Exp & 0,00 & 0,88 & 0,88 & 46,30 & 138,70 & -0.0065 & 0.8086 & $-0,0043$ & 0,9542 \\
DFD Oeste & OLS - C & Exp & 0,14 & 0,96 & 1,10 & 63,81 & 191,15 & -0.0095 & 0.8924 & -0.0055 & 0.9838 \\
\hline
\end{tabular}

$\mathrm{C}_{0}$ - efeito pepita; $\mathrm{C}_{1}$ - contribuição; $\mathrm{C}_{0}+\mathrm{C}_{1}$ - patamar; a - alcance; a' - alcance prático; EM - erro médio; $\mathrm{DP} \mathrm{EM}_{-}$desvio-padrão do erro médio; $\mathrm{ER}$ - erro médio reduzido; $\mathrm{S}_{\mathrm{ER}}$ desvio-padrão do erro médio reduzido; $\mathrm{ML}$ - máxima verossimilhança; REML - máxima verossimilhança restrita; OLS - C - mínimos quadrados ordinário estimador clássico; WLS - R - mínimos quadrados ponderados estimador clássico; WLS - R - mínimos quadrados ponderados estimador robusto; Esf - esférico; Exp - exponencial.

Os resultados da validação permitiram escolher os melhores ajustes (os critérios de escolha baseados na validação são: escolha das combinações em que os valores de EM e de ER são mais próximos de zero, em que o valor $\mathrm{DP}_{\mathrm{EM}}$ seja o menor possível, e em que o valor de $\mathrm{S}_{\mathrm{ER}}$ que seja o mais próximo de um). Assim, os valores escolhidos estão na Tabela 2. 

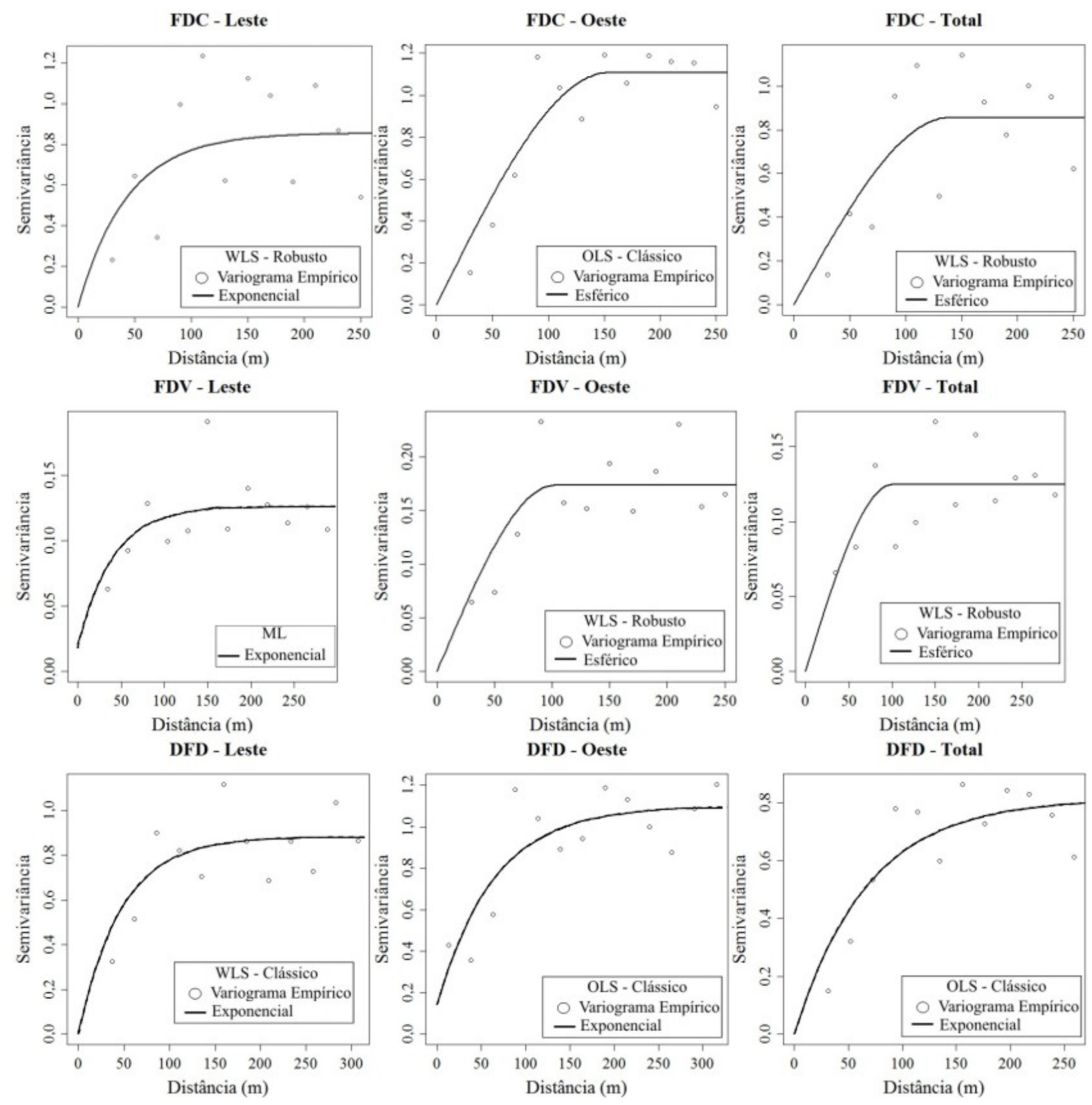

FIGURA 2. Semivariogramas escolhidos em função do método e modelo para a força de desprendimento de frutos cereja (FDC) e verde (FDV), e da diferença da força de desprendimento (DFD) na face de exposição solar leste, oeste ou independentemente da face de exposição. Semivariograms chosen based on the methods and models to the detachment force of mature fruit (FDC) and green fruit (FDV), and the difference between the detachment force (DFD) according to the solar side exposure east, west or independent of the exposure face.

O modelo esférico ajustou-se para a função semivariograma de quatro variáveis (FDC Total, FDC Oeste, FDV Oeste e FDV Total), sendo que, para as demais variáveis, a função semivariograma teve como melhor ajuste o modelo exponencial. O modelo matemático esférico pode ser visto em outros trabalhos de estudo de variabilidade espacial em lavouras cafeeiras de atributos do solo, produtividade, desfolha e infestação de praga (ALVES et al., 2009a; MOLIN et al., 2010; SILVA et al., 2007; SILVA et al., 2008; SILVA et al., 2010a; SILVA et al., 2010c). ALVES et al. (2009b), estudando variabilidade espacial de doenças de uma lavoura cafeeira, ajustaram o modelo exponencial para a função semivariograma. SILVA et al. (2010a) também 
utilizaram o modelo exponencial para ajustar a função semivariograma para alguns atributos do solo.

O efeito pepita é um parâmetro utilizado para explicar duas fontes de variação: variação do atributo para distâncias menores que a considerada na grade de amostragem, e erros de medição. Foram encontrados valores de efeito pepita diferentes de zero apenas para FDV Leste e para DFD Oeste, sendo 0,17 e 0,14, respectivamente.

Os valores do alcance dos semivariogramas têm uma importância considerável na determinação do limite da dependência espacial, ou seja, é de fundamental importância para o conhecimento do real alcance da dependência espacial da variável em estudo. As variáveis estudadas apresentaram diferentes alcances de dependência espacial, sendo que a variável FDC Total apresentou alcance igual a 139,51 m, a FDC Leste foi igual a 129,35 m e a FDC Oeste foi de 154,18 m. Para a FDV Total, foi obtido o alcance igual a 99,92 m, para a FDV Leste foi de 98,94 m e para FDV Oeste obteve-se o alcance igual a 102,32 m.

Posteriormente, foram estimados, por krigagem ordinária, tendo como base a dependência espacial dos modelos de semivariogramas, os valores da FDC Leste, FDC Oeste, FDC Total, FDV Leste, FDV Oeste, FDV Total, DFD Leste, DFD Oeste e DFD Total. Sabe-se da teoria geoestatística que a Krigagem apresenta as melhores estimações dos valores não amostrados, no sentido de não tendenciosidade e de erro quadrático médio, isto é, as estimativas dos valores da variável nos locais não amostrados, são, em média, não tendenciosas e com erro quadrático o menor possível dentre os estimadores lineares (OLIVEIRA, 1991). Esta propriedade da krigagem depende, é claro, para ser completamente verificada, de um bom ajuste de modelos de semivariogramas. Assim, devido à obtenção dos melhores ajustes por validação, conforme apresentado na Tabela 2, foi possível confeccionar mapas de distribuição espacial para todas as variáveis (Figura 3), que permitiram visualizar a variabilidade espacial da força de desprendimento.

Analisando-se os mapas de força de desprendimento dos frutos cerejas, apresentados na Figura 3, verifica-se que estes possuem o mesmo padrão de distribuição com relação à face de exposição solar, ou seja, se a face leste apresenta elevada força de desprendimento, o mesmo ocorre na face oeste, o que se reflete no mapa de exposição em área total da planta. Este mesmo comportamento pode ser percebido na força de desprendimento de frutos verdes.

A eficiência de derriça está relacionada com a carga pendente da planta bem como com número de frutos na roseta (SANTOS et al., 2010); portanto, o sentido do caminhamento da colhedora em campo não afeta as variáveis analisadas. Dependendo da topografia da lavoura e/ou de fatores econômicos do produtor, irá optar-se pelo melhor modelo de máquina que irá atender satisfatoriamente à operação de colheita. Colhedoras com um cilindro operam em regiões de topografia mais acidentada que as de dois cilindros (SILVA, 2004), sendo que esta última apresenta um custo de aquisição superior ao da primeira (SILVA et al., 2012).

Na colheita manual seletiva, os cafeicultores podem escolher quais frutos desejam coletar, escolhendo aqueles que estiverem na maturação ótima para a colheita. Para a realização da colheita seletiva mecanizada, passa-se a colhedora ao menos duas vezes na área (ou seja, em duas épocas), em função da quantidade de frutos verdes e cerejas existentes nas plantas (OLIVEIRA et al., 2007a; OLIVEIRA et al., 2007b; OLIVEIRA et al., 2007c). OLIVEIRA et al. (2007b), comparando os custos da operação de colheita, observaram que a colheita seletiva mecanizada (duas passadas da colhedora) apresentou a redução de $62,4 \%$ dos custos com relação à colheita manual total. OLIVEIRA et al. (2007c), estudando a influência da colheita mecanizada na produção cafeeira, observaram que a produtividade da lavoura cafeeira não foi influenciada pela colheita mecanizada, seja ela seletiva (duas passadas), seja não seletiva (uma passada), ao longo dos anos estudados.

Porém, quando se realiza a colheita mecanizada, pode ser difícil realizar o processo seletivo, mesmo realizando-se as duas passadas da colhedora, pois muitos frutos verdes ainda podem ser coletados junto aos frutos cerejas. De acordo com BORÉN (2008), a maturação do fruto de cafeeiro 
está diretamente relacionada com a humidade. Este autor ainda afirma que a humidade é a maior responsável pela perda de qualidade do produto, uma vez que ela controla o processo de fermentação e o potencial desenvolvimento de fungos durante o armazenamento e o transporte, 0 que pode resultar em sabor e aroma pobres a partir das toxinas produzidas.

Desta maneira, visando a melhorar a eficiência da colheita seletiva mecanizada, pode-se utilizar a força de desprendimento dos frutos de cafeeiro. SILVA (2008) cita que a força de desprendimento dos frutos pode ser utilizada como um parâmetro objetivo, principalmente para os frutos cerejas, por indicar o momento de se iniciar a colheita mecanizada e a seletiva do café. SILVA et al. (2010b) citam que, quanto maior a diferença entre a força de desprendimento dos frutos verdes e cerejas, melhor será a colheita mecanizada seletiva dos frutos do cafeeiro.

Desta forma, os mapas de isolinhas da diferença entre a força de desprendimento, semelhantes aos apresentados neste trabalho, podem ser utilizados pelos cafeicultores para regular suas colhedoras de forma a permitir a colheita seletiva dos frutos. Tais mapas ainda podem ser utilizados na escolha do melhor momento e os melhores locais para se iniciar a colheita mecanizada e a seletiva do café, o que pode melhorar a qualidade do produto final que, por sua vez, resultará em aumento da lucratividade.

A partir da análise dos mapas da força de desprendimento dos frutos do cafeeiro (Figura 3), observou-se que o melhor local para iniciar a colheita seria a área central, que apresentou a menor força de desprendimento dos frutos cerejas e uma grande diferença entre a força de desprendimento entre frutos verde e cereja. 

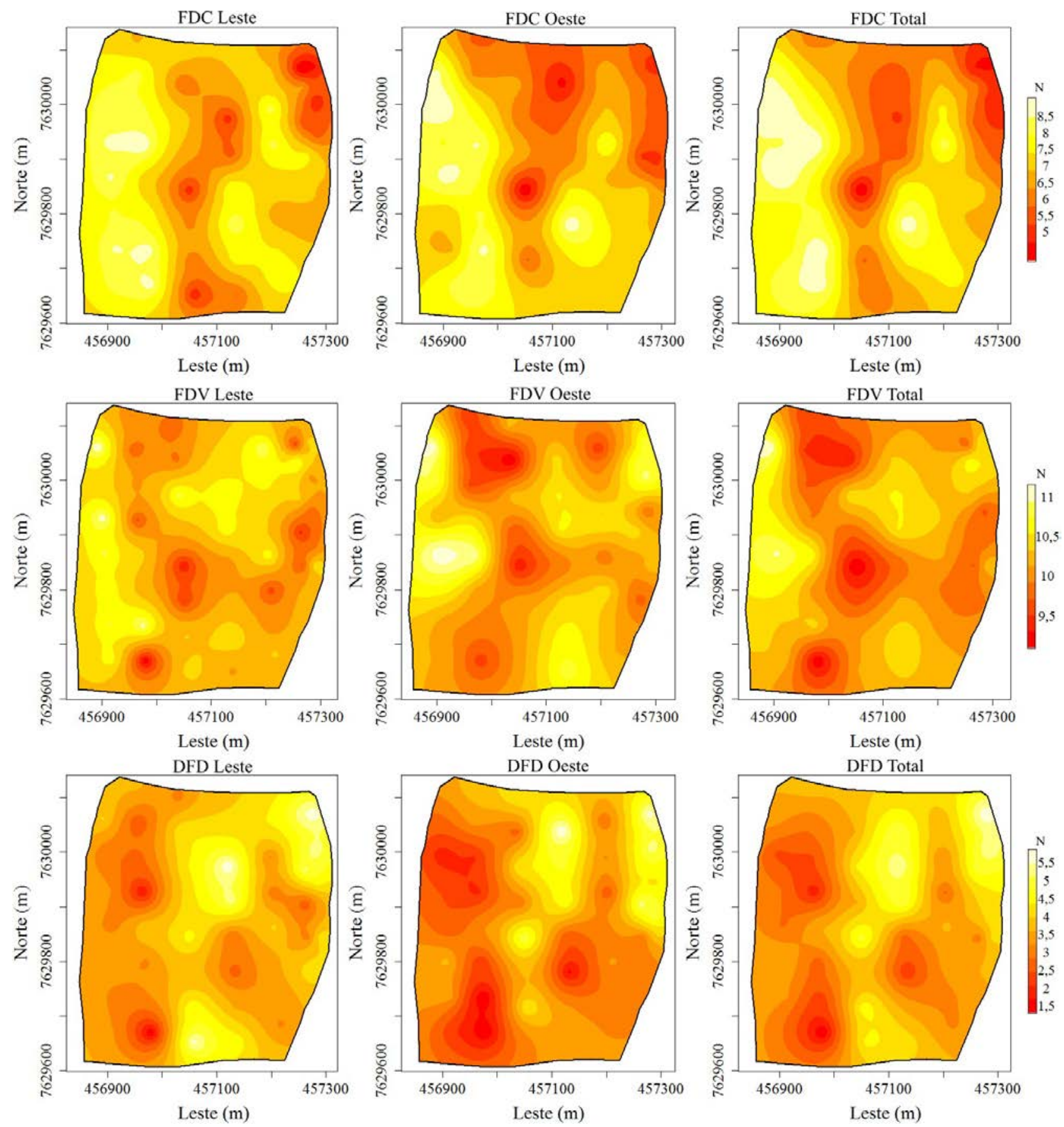

FIGURA 3. Distribuição espacial da força de desprendimento de frutos cereja (FDC) e verde (FDV) na face leste, oeste e em área total, e também da diferença da força de desprendimento entre os frutos verde e cereja (DFD), independentemente da face de exposição solar (total), no lado leste e oeste do cafeeiro, no ano de 2009. Spatial distribution of detachment force of mature (FDC) and green fruit (FDV) on the east and west face and in the hole area and as well as the difference between the detachement force of green and mature fruit (DFD) independent of the solar exposure side (total), in the east side and in the west side of the coffee plant in 2009. 


\section{CONCLUSÕES}

As variáveis em estudo (força de desprendimento de frutos cerejas - FDC, força de desprendimento de frutos verdes - FDV, e a diferença entre estas forças - DFD) de acordo com a face de exposição solar (Leste e Oeste) e em área total de exposição, no ano de 2009, apresentam estruturas de dependência espacial, modeladas pelos semivariogramas, obtidos pelas estatísticas de validação.

Por meio da análise dos mapas de isolinhas da distribuição espacial das variáveis em estudo, obtidos por krigagem, é possível identificar os locais mais propícios para se iniciar a colheita seletiva e mecanizada dos frutos do cafeeiro.

\section{REFERÊNCIAS}

ALVES, M. C.; SILVA, F. M.; MORAES, J. C.; POZZA, E. A.; OLIVEIRA, M. S.; SOUZA, J. C. S.; ALVES, L. S. Geostatistical analysis of the spatial variation of the berry borer and leaf miner in a coffee agroecosystem. Precision Agriculture, Dordrecht, v. 10, n. 12, p. 1-14, Dec. 2009a.

ALVES, M. C.; SILVA, F. M.; POZZA, E. A.; OLIVEIRA, M. S. Modeling spatial variability and pattern of rust and brown eye spot in coffee agroecosystem. Journal of Pest Science, Berlin, v. 82, n. 2, p. 137-148, May 2009b.

BACHMAIER, M.; BACKES, M. Variogram or semivariogram? Understanding/the variances in a variogram. Precision Agriculture, Dordrecht, v 9, p. 173-175. 2008.

BORÉN, F. M. A água nos frutos e nos grãos de café. In: BORÉN, F.M. (Ed.) Pós-colheita do café. Lavras: Universidade Federal de Lavras, 2008. p.127-158.

CARVAlHO, G. R.; BOTELHO, C. E.; BARTHOLO, G. F.; PEREIRA, A. A.; NOGUEIRA, Â. M.; CARVALHO, A. M. de. Comportamento de progênies F4 obtidas por cruzamentos de 'Icatu' com 'Catimor'. Ciência e Agrotecnologia, Lavras, v. 33, n. 1, p. 47-52, jan./fev. 2009.

CRESSIE, N. Statistics for spatial data. New York: J. Wiley, 1993. 900 p.

CRESSIE, N.; HAWKINS, D. M. Robust estimation of the variogram. Mathematical Geology, New York, v. 12, n. 2, p. 115-125, 1980.

FARACO, M. A., URIBE-OPAZO, M. A., SILVA, A. A., JOHANN, J. A., BORSSOI, J. A. Seleção de modelos de variabilidade espacial para elaboração de mapas temáticos de atributos físicos do solo e produtividade da soja. Revista Brasileira de Ciência do Solo, Viçosa, MG, v. 32, n. 2, p. 463-476, mar./abr. 2008.

FERRAZ, G. A. S.; SILVA, F. M. CARVALHO, L. C. C.; ALVES, M. C.; FRANCO, B. C. Variabilidade espacial e temporal do fósforo, potássio e da produtividade de uma lavoura cafeeira. Engenharia Agrícola, Jaboticabal, v.32, n.1, p.140-150, jan./fev. 2012.

ISAAKS, E. H.; SRIVASTAVA, R. M. An introduction to applied geostatistics. New York: Oxford University, 1989. $561 \mathrm{p}$.

JOHANN, J. A.; SILVA, M. C. A.; URIBE-OPAZO, M. A.; DALPOSSO, G. H. Variabilidade espacial da rentabilidade, perdas na colheita e produtividade do feijoeiro. Engenharia Agrícola, Jaboticabal, v. 30, n.4, p. 700-714, jul/ago. 2010.

LOPES, P. R.; ARAÚJO, K. C. S.; FERRAZ, J. M. G.; LOPES, I. M.; FERNANDES, L. G. Produção de café agroecológico no sul de Minas Gerais: sistemas alternativos à produção intensiva em agroquímicos. Revista Brasileira de Agroecologia,Porto Alegre, v. 7, n.1, p. 25-38, 2012

MINGOTI, S. A.; ROSA, G. A note on robust and non-robust variogram estimators. REM: R. Esc. Minas, Ouro Preto, v. 61, n.1. p.87-95, 2008. 
MOLIN, J. P.; MOTOMIYA, A. V. A.; FRASSON, F. R.; FAULIN, G. Di C.; TOSTA, W. Teste procedure for variable rate fertilizer on coffee. Acta Scientiarum Agronomy, Maringá, v.32, n.4, p. 569-575, 2010.

OLIVEIRA, E; SILVA, F. M.; SALVADOR, N.; FIGUEIREDO, C. A. P. Influência da vibração das hastes e da velocidade de deslocamento da colhedora no processo de colheita mecanizada do café. Engenharia Agrícola, Jaboticabal, v.27, n.3, p.714-721, 2007a.

OLIVEIRA, E; SILVA, F. M.; SALVADOR, N.; SOUZA, Z. M.; CHALFOUN, S. M.; FIGUEIREDO, C. A. P. Custos operacionais da colheita mecanizada do cafeeiro. Pesquisa Agropecuária Brasileira, Brasília, v.42, n.6, p.827-831, 2007b.

OLIVEIRA, E; SILVA, F. M.; SOUZA, Z. M.; FIGUEIREDO, C. A. P. Influência da colheita mecanizada na produção cafeeira. Ciência Rural, Santa Maria, v.37, n.5, p.1466-1470, 2007c.

OLIVEIRA, M. S. Planos amostrais para variáveis espaciais utilizando Geoestatística. 1991. 100 f. Dissertação (Mestrado em Estatística) - Universidade Estadual de Campinas, Campinas, 1991.

ORTEGA, A. C.; JESUS, C. M. Território café do Cerrado: transformações na estrutura produtiva e seus impactos sobre o pessoal ocupado. Revista de Economia e Sociologia Rural, Brasília, v.49, n.3, p. 771-800, 2011.

RIBEIRO JUNIOR, P. J.; DIGGLE, P. J. GeoR: a package for geostatistical analysis. R-News, New York, v. 1, n. 2, p. 14-18, 2001.

RIBEIRO, M. S.; LIMA, L. A.; FARIA, F. H. S.; REZENDE, F. C.; FARIA, L. A. Efeitos de águas residuárias de café no crescimento vegetativo de cafeeiros em seu primeiro ano. Engenharia Agrícola, Jaboticabal, v.29, n.4, p.569-577, 2009.

SANTOS, F. L.: QUEIROZ, D. M.: PINTO, F. A. C.; RESENDE, R. C. Efeito da frequência e amplitude de vibração sobre a derriça de frutos de café. Revista Brasileira de Engenharia Agrícola e Ambiental, Campina Grande, v.14, n.4, p.425-431, 2010.

SILVA, A. F.; LIMA, J. S. S.; SOUZA, G. S.; OLIVEIRA, R. B. Variabilidade espacial de atributos químicos do solo cultivado com café arábica (Coffea arabica l.) sob diferentes manejos. Coffee Science, Lavras, v. 5, n. 2, p. 173-182, 2010a.

SILVA, F. C. Efeito da força de desprendimento e maturação dos frutos de cafeeiros na colheita mecanizada. 2008. 106 p. Dissertação (Mestrado em Engenharia Agrícola) - Universidade Federal de Lavras, Lavras, 2008.

SILVA, F. C.; SILVA, F. M.; ALVES, M. C.; BARROS, M. M.; SALES, R. S. Comportamento da força de desprendimento dos frutos do cafeeiro ao longo do período da colheita. Ciência e Agrotecnologia, Lavras, v.34, 468-474, 2010 b.

SILVA, F. C.; SILVA, F. M.; FERRAZ, G. A. S.; RAMOS, M. C.; SILVA, E. P. Comparativo de duas metodologias para obtenção do custo horário de máquinas na colheita mecanizada do café. In: X CONGRESO LATINOAMERICANO Y DEL CARIBE DE INGENIERÍA AGRÍCOLA, 10.; CONGRESSO BRASILEIRO DE ENGENHARIA AGRÍCOLA, 48., 2012. Anais... Londrina: ALIA/SBEA, 2012.

SILVA, F. M. Colheita mecanizada e seletiva do café: cafeicultura empresarial: produtividade e qualidade. Lavras: Ufla/Faepe, 2004. 75p.

SILVA, F. M.; ALVES, M. C.; SOUZA, J. C. S.; OLIVEIRA, M. S. Efeitos da colheita manual na bienalidade do cafeeiro em Ijaci, Minas Gerais. Ciência e Agrotecnologia, Lavras, v. 34, n. 3, p. 625-632, 2010c.

SILVA, F. M.; ARRÉ, T. J.; TOURINO, E. S.; GOMES, T. S.; ALVES, M. C. USO DE ETHREL NA COLHEITA MECANIZADA E SELETIVA DE CAFÉ ARÁBICA (Coffea arabica L.). Coffee Science, Lavras, v. 4, n. 2, p. 178-182, 2009. 
SILVA, F. M.; SILVA, F. C.; SILVA, A. C. R.; ALVES, M. C.; SOUZA, J. C. S. Influência da força de desprendimento dos frutos do cafeeiro em diferentes estádios de maturação ao longo da colheita. In: CONGRESSO BRASILEIRO DE ENGENHARIA AGRÍCOLA, 36., 2007, Bonito. Anais... Jaboticabal: SBEA, 2007.

SILVA, F. M.; SOUZA, Z. M.; FIGUEIREDO, C. A. P.; MARQUES, J. J.; MACHADO, R. V. Variabilidade espacial de atributos químicos e de produtividade na cultura do café. Ciência Rural, Santa Maria, v.37, n. 2, p.401-407, 2007.

SILVA, F. M.; SOUZA, Z. M.; FIGUEIREDO, C. A. P.; VIEIRA, L. H. S.; OLIVEIRA, E. Variabilidade espacial de atributos químicos e produtividade da cultura do café em duas safras agrícolas. Ciência e Agrotecnologia, Lavras, v.32, n.1, p.231-241, 2008.

SOUZA, C.M.A.; QUEIROZ, D.M.; PINTO, F.A.C.; RAFULL, L.Z.L. Desenvolvimento de uma máquina vibradora para estudo do desprendimento de frutos do cafeeiro. Engenharia Agrícola, Viçosa, MG, v.13, n.1, p.7-18, 2005.

WEBSTER, R.; OLIVER, M. Geostatistics for environmental scientists. Chichester: John Wiley \& Sons, 2007. 